\title{
Construcción de un índice de satisfacción para clientes de supermercados mexiquenses Una investigación exploratoria
}

\author{
Pilar Arroyo López* \\ Lorena Carrete Lucero* \\ Sara Isabel García López Legorreta*
}

\begin{abstract}
Resumen
La satisfacción del cliente es uno de los conceptos de mercadotecnia de interés tanto para académicos como para profesionales debido a que prácticamente todas las actividades de la empresa pueden ser evaluadas en términos de su contribución a la satisfacción del cliente. El desarrollo de índices de satisfacción requiere de una comprensión, interpretación y operacionalización cuidadosa del concepto para que resulte una medida útil para la empresa. La relevancia del tema motiva la realización de este trabajo cuyo objetivo es elaborar y apoyar empíricamente la validez de un índice de satisfacción para el cliente de supermercados mexiquenses.

Una encuesta aplicada entre los clientes de una cadena de supermercados de cobertura regional aporta los datos necesarios para realizar un análisis factorial a través del cual se verifica la multidimensionalidad del concepto de satisfacción, identificándose siete dimensiones latentes al concepto, las cuales apoyan la validez convergente de la multiescala elaborada. Un análisis de regresión posterior, en el cual la lealtad intencional del cliente figura como variable dependiente, apoya la validez predictiva del índice y permite identificar aquellos componentes de satisfacción que tienen una influencia significante en la lealtad del cliente de supermercados.
\end{abstract}

Palabras clave: satisfacción del consumidor, lealtad, supermercados, validez, multidimensionalidad.

Fecha de recepción: 10/08/2007

Fecha de aceptación: 06/12/2007

*Profesoras del Instituto Tecnológico y de Estudios Superiores de Monterrey, Campus Toluca.

Correos electrónicos: pilar.arroyo@itesm.mx, 1carrete@itesm.mx, sarai.garcia@itesm.mx

No. 225, mayo-agosto 2008: 59-78 
Pilar Arroyo López, Lorena Carrete Lucero y Sara Isabel García López Legorreta

\title{
Development of a satisfaction index for Mexican supermarkets customers. An exploratory research
}

\begin{abstract}
Customer satisfaction is one on the concepts of interest to both practitioners and researchers because almost all activities of the organization can be evaluated in terms of their contribution to customer satisfaction. Then the development of satisfaction measures requires of a careful comprehension, interpretation and operationalization of the satisfaction concept so the resulting measures can really be useful to the organization. The need to provide suitable satisfaction measures is the main motivation for this work, whose main objective is to develop and provide empirical evidence for the validity of a satisfaction index for the customer of Mexican supermarkets. A survey among the customers of a supermarket chain with regional coverage supplies the required data to conduct a factor analysis that permits to verify the multidimensionality of the satisfaction concept and to identify seven underlying latent dimensions for the construct whose structure gives evidence of convergent validity. A posterior regression analysis with intentional loyalty as the dependent variable supports the predictive validity of the satisfaction index and allows the identification of those satisfaction's components with a significant influence on the loyalty of the supermarket's clients.
\end{abstract}

Key words: customer satisfaction, loyalty, supermarkets, validity, multidimensionality.

\section{Introducción y marco teórico}

$\mathrm{E}^{\mathrm{l}}$ 1 sector supermercado constituye un importante eslabón del sistema productivo mexicano porque es el canal de distribución de una gran cantidad de bienes. Esta industria muestra una expansión en las últimas décadas, principalmente como resultado del crecimiento de la economía nacional, lo cual se refleja en el aumento de la capacidad de compra del consumidor, del desarrollo de distintos formatos para los diferentes niveles socioeconómicos de la población y de la incorporación de servicios que no se ofrecían tradicionalmente en estos establecimientos, como es el caso de una mayor oferta de créditos por parte de los detallistas, aceptación de nuevas formas de pago, ampliación de horarios y entrega de productos a domicilio.

Las empresas que venden al menudeo contribuyen a crear valor para el consumidor al atender sus necesidades y facilitar la disponibilidad de productos en el lugar y tiempo correctos. La industria de venta al menudeo en la actualidad incluye a una gran variedad de negocios (retailers) que van desde grandes cadenas con múltiples unidades de negocios hasta pequeños locales operados por individuos emprendedores. De acuerdo con datos publicados por el INEGI, el índice de ventas netas de las tiendas de autoservicio, incluidos los supermercados, registró un crecimiento pro- 
Construcción de un índice de satisfacción para clientes de supermercados mexiquenses

Una investigación exploratoria

medio de 7.15\% en los últimos cinco años (periodo 2001 al 2006). La Asociación Nacional de Tiendas de Autoservicio y Departamentales (ANTAD) reporta igualmente un incremento en las ventas de autoservicios de enero a abril de 2007 del 7.2\%, que está influido - entre otros factores- por el crecimiento en las ventas de supermercado (abarrotes y perecederos), el cual ascendió a un 7.0\% (Boletín de Prensa de la ANTAD, 2007). Adicionalmente, el Food Marketing Institute de Estados Unidos (FMI) y la ANTAD indican que los supermercados aparecen como el establecimiento preferido de los mexicanos para comprar alimentos (con 57\% de las preferencias), le siguen los mercados establecidos (25\%), las tiendas de abarrotes (9\%), los hipermercados $(4 \%)$ $\mathrm{y}$ los mercados sobre ruedas (3\%).

La importante contribución de la industria de venta al menudeo para la economía y el empleo del país, así como su papel crítico como punto de contacto entre el consumidor y los fabricantes ha despertado el interés por su estudio. El impacto de factores macroambientales (e.g. estado de la economía, aspectos culturales, avances tecnológicos) sobre el desarrollo de la industria, las características y resultados de las estrategias de colaboración entre detallistas y productores (e.g. ECR y CPFR), la importancia de introducir los elementos necesarios - no sólo para completar la transacción compra-venta sino para crear una experiencia de compra-, la aportación del detallista a la formación de una imagen propia y para los productos del fabricante, son sólo algunos de los aspectos desde los cuales la academia ha abordado la investigación de la industria de venta al menudeo. Revistas académicas especializadas como el Journal of Retailing y el International Journal of Retail and Distribution Management, además de libros técnicos como Retailing Management (Mc Graw Hill, 2007), Retailing (MacMillan, 2003), Retail Marketing Management (Prentice-Hall, 1999) y Retail Marketing (McGraw Hill, 1990), por citar algunos, reportan los avances en el estudio de este tema y estructuran el área identificando las funciones básicas de la industria, sus estrategias de mercadotecnia y abasto, así como las actividades operativas que los detallistas realizan para asegurar la disponibilidad de productos.

Este estudio se ubica en el contexto de cómo el detallista es evaluado por parte del consumidor en relación con la satisfacción experimentada con la prestación del servicio. El interés en este tema es debido a que se reconoce que el desempeño financiero de las organizaciones está relacionado con la satisfacción de su clientela. Kristensen et al. (2001) reportan que un incremento del $1 \%$ en la satisfacción de los clientes produce un incremento de $0.4 \%$ en el ROI y un incremento del $7 \%$ en el valor para los accionistas (shareholders'value). En virtud de estos hallazgos - y dado que la mayor parte de la investigación en el sector detallista se ha enfocado a las grandes cadenas de venta al menudeo con cobertura mundial, o se ha realizado en el contexto de países desarrollados con una industria de venta al menudeo madura y competitiva- se 
identifica la necesidad de complementar el conocimiento sobre el comportamiento poscompra del consumidor mexicano y llevarlo al contexto local no sólo para verificar la aplicabilidad de los resultados obtenidos en otros entornos, sino también para comprender la evolución en las preferencias y necesidades de los consumidores a medida que más sitios de compra les son accesibles y los detallistas compiten por atraerlos. El propósito de este trabajo es desarrollar un índice de satisfacción para clientes de supermercados de cobertura regional y analizar su relación con sus intenciones de lealtad, considerada esta última como un recurso con el que cuentan las organizaciones para asegurar su rentabilidad y su participación en el mercado.

En relación con los factores que se identifican en la literatura como determinantes de la satisfacción en este sector de actividad, se tienen los precios competitivos, la calidad y variedad de la mercancía, la ubicación conveniente del establecimiento, los servicios requeridos para facilitar el proceso de compra y la asistencia de los empleados. La percepción de los precios de la tienda con respecto de los precios de la competencia es una de las variables que influye en la percepción de valor para el cliente $y$, por ende, es un componente crítico que incide en la satisfacción y en la decisión de continuar favoreciendo al establecimiento para realizar sus compras (Sweeney y Soutar, 2001). Los supermercados en ese sentido se han abocado a mejorar sus actividades operativas a efecto de reducir sus costos y con ello apoyar sus estrategias de precios bajos y amplio surtido.

Si bien el precio es un factor de peso en la satisfacción del consumidor, éste no es el único componente que incide en la complacencia del cliente con su compra en cierto supermercado y con su comportamiento poscompra, en particular la compra repetida. Fornell et al. (1996) autores del ACsI (Índice Nacional de Satisfacción del Consumidor Americano) y Kristensen et al. (2001) autores del ECSI (Índice Nacional de Satisfacción del Consumidor Europeo) concluyen que la oferta de una mezcla de surtidos - tanto en calidad como en variedad - acorde con las necesidades del cliente es otro elemento crítico para la satisfacción. Los atributos de la tienda (como ubicación, horarios de apertura y tiempo de espera en cajas) y los servicios complementarios (como formas de pago aceptadas y servicio de estacionamiento) también se reportan como componentes importantes de la satisfacción del cliente de supermercados (Gail y Scott 1995; McDonald, 1991; Chang y Tu, 2005).

La satisfacción se ve además influida por otros elementos no tangibles como es la asistencia de los empleados del establecimiento con los que el cliente interactúa. La actitud y la competencia del personal ha sido estudiada por autores como Kristensen et al. (2001) en su medición de los niveles de satisfacción, concluyendo que el servicio del personal es un componente que puede influir de manera contundente en 
Construcción de un índice de satisfacción para clientes de supermercados mexiquenses

Una investigación exploratoria

el juicio de satisfacción del cliente. Spreng et al. (1996) y Al-Awadi (2002) también hacen referencia a la importancia de la capacidad del personal de ventas para dar solución a las dudas o problemas, lo que influye positivamente en la satisfacción de los consumidores.

Aunque la dinámica de satisfacción del consumidor ha sido poco estudiada en relación con los diferentes formatos de establecimiento (tiendas de descuento, supermercados, hipermercados, tiendas tradicionales), los supermercados muestran los mayores índices de satisfacción (Kristensen et al. 2001) en particular por el efecto de la imagen de estos detallistas. Las variables de imagen de los supermercados que más inciden en la satisfacción del cliente son la creatividad, la orientación al mercado y la confiabilidad que los clientes perciben para este tipo de formato de establecimiento de venta al menudeo.

El interés por la medición de la satisfacción radica en las respuestas o consecuencias que puede generar, en particular se enfatiza el impacto que la satisfacción tiene sobre el comportamiento de lealtad y el comportamiento de queja del consumidor. De acuerdo con el modelo del ACSI (Fornell et al., 1996), entre más satisfecho se encuentra un cliente mayor es la probabilidad de que se incremente su lealtad y menor la probabilidad de que presente una queja; se encuentra sobre todo evidencia empírica del efecto negativo que la insatisfacción tiene sobre la lealtad. Se han elaborado múltiples propuestas de conceptualización de lealtad, lo que resulta en diferentes formas de operacionalización para el concepto. Fornell et al. (1996), por ejemplo, miden la lealtad a través de la recompra y la tolerancia del consumidor a cambios en los precios. Bloemer y Ruyter (1997) operacionalizan la lealtad a partir de la intención de recompra del cliente y del compromiso con el producto/servicio o negocio. Por su parte, Oliver (1999) propone un modelo conceptual que presenta a la lealtad como un proceso en varias etapas. Éste inicia con una lealtad cognitiva (evaluar positivamente el desempeño de la marca); continúa con una lealtad afectiva (sentirse bien con la marca) y una lealtad conductual (intención de recomprar la marca) y culmina con una lealtad acción (recompra de la marca). Sawmong y Omar (2004) retoman estas etapas para evaluar el nivel de lealtad de los consumidores del sector supermercado, estableciendo que la lealtad cognitiva y afectiva son influidas por aspectos ya antes citados como son la percepción sobre precio competitivo, la calidad de los productos, la comodidad para comprar con niños y las formas de pago aceptadas. Otros estudios confirman la incidencia de la calidad del servicio y de la mercancía en la lealtad del consumidor de este sector (Sirohi et al. 1998). 


\section{Planteamiento del problema}

Si bien la satisfacción del cliente es un constructo de notable importancia no sólo para el sector de supermercados, dada su incidencia en la rentabilidad de las organizaciones, la evaluación del concepto es difícil debido tanto a su complejidad como a que dependa de las percepciones y actitudes de los consumidores. El objetivo de este trabajo es elaborar un índice de satisfacción y proporcionar apoyo empírico para la multidimensionalidad y validez de la medida propuesta. Específicamente, el índice de satisfacción se desarrolla para clientes de supermercados mexiquenses y su estructura a priori se define con base en la revisión de la bibliografía especializada. Para apoyar la validez predictiva del índice elaborado, se incorpora el concepto de lealtad desde la consideración de que la satisfacción es un antecedente de las intenciones de lealtad de los consumidores. La selección del sector supermercado obedece a su importancia económica y la alta competencia a la que está sujeto, lo que hace valioso incorporar una medida de satisfacción al conjunto de métricas de desempeño que utilizan los supermercados.

\section{Metodología}

La primera etapa de la metodología fue el diseño de un conjunto de reactivos que aseguraran la validez de contenido del índice. Para ello, se tomaron en cuenta cuatro componentes de la satisfacción que en forma recurrente se citan en la literatura: competitividad de los precios del supermercado (Sirohi et al., 1998), percepción sobre el diseño y los servicios básicos que ofrece el establecimiento (Gail y Scott, 1995; McDonald, 1991; Sawmong y Omar, 2004; Chang y Tu, 2005), servicio y atención que prestan los empleados (Sirohi et al., 1998; Sawmong y Omar, 2004) y conveniencia y calidad de la mercancía (Sirohi et al., 1998, McDonald, 1991). Para cada uno de estos componentes de satisfacción se elaboró un cierto número de reactivos cuyo contenido se apoyó en los estudios correspondientes para medición de satisfacción que se identificaron en la revisión de trabajos precedentes realizados en otros contextos y que involucran al sector de venta al menudeo.

Para los reactivos referentes al componente mercancía en la tienda, se utilizó una escala ordinal de cinco categorías, en la cual las anclas son $1=$ excelente a $5=$ pésima. Los reactivos de los otros tres componentes están sobre una escala Likert que va de $1=$ totalmente de acuerdo, a 5 = totalmente en desacuerdo. Para no forzar al cliente del supermercado a expresar un juicio cuando no ha requerido de ciertos servicios (por ejemplo, entrega a domicilio) o no ha notado determinados atributos de la tienda (intervenciones del gerente) se incluyó una categoría adicional etiquetada como "no sabe". 
Construcción de un índice de satisfacción para clientes de supermercados mexiquenses

Una investigación exploratoria

Los reactivos que constituyen cada uno de los cuatro componentes de satisfacción fueron integrados a un cuestionario que en total incluye 45 preguntas organizadas en siete secciones. La primera sección (A) investiga sobre las preferencias del cliente en materia de supermercados, su frecuencia de compra y su satisfacción global con el supermercado en el cual se aplicó la encuesta. Estas preguntas no sólo proporcionan información general del comportamiento de los consumidores, sino que también sirvieron para asegurar que los respondientes fueran clientes del supermercado, lo que garantiza que han sido expuestos a múltiples experiencias de compra y, por tanto, son sujetos apropiados para expresar su satisfacción con el servicio del establecimiento.

La sección dos (в) fue nombrada como percepción de valor y contiene tres reactivos que refieren a la relación precio/servicio y al precio de los productos con relación a los de la competencia. La sección tres (c) es la más amplia, incluye 16 reactivos que consideran aspectos de diseño de las instalaciones y la evaluación de los servicios de apoyo tales como horarios, formatos de pago, estacionamiento, etc. La ubicación del supermercado, elemento individual que la alta gerencia de los supermercados y la literatura (Sawmong y Omar, 2004; McDonald, 1991) citan como crítico para la preferencia y satisfacción del consumidor se incluyó en esta sección. La cuarta sección (D) tiene ocho reactivos, los cuales abordan las percepciones sobre la competencia y la atención de empleados y gerente de la tienda. La sección cinco (E) cuenta con cinco reactivos que cuestionan sobre la calidad, variedad y surtido de la mercancía. La sección seis (F) está formada por tres reactivos que evalúan las intenciones de lealtad de los clientes, estos reactivos están sobre una escala de probabilidad de exhibir ciertas conductas. La escala cuenta con cinco categorías que van desde 1 = seguro que sí a $5=$ seguro que no (realizará cierta acción). Una pregunta abierta fue incluida al final de esta sección para que el cliente expresara algún comentario particular con respecto del establecimiento. La última sección (G) contiene cuatro reactivos que exploran el perfil demográfico y económico del cliente. Esta sección fue incluida para verificar la representatividad de la muestra obtenida y no con fines para realizar comparaciones entre los grados de satisfacción, según el perfil del cliente.

El instrumento de medición fue revisado por los gerentes de diez unidades de una cadena de supermercados local (la unidad de análisis seleccionada) que lo juzgaron apropiado para los fines del estudio. Este juicio, sumado a la revisión de la literatura en la que se basó su diseño y la revisión cruzada que hicieron los investigadores de este proyecto, aseguran la validez facial del índice propuesto. Antes de realizar la encuesta, los responsables de este trabajo aplicaron el cuestionario a quince consumidores. Esta preprueba permitió corregir la sintaxis de los reactivos, modificar algunos términos que no resultaban claros al respondiente (por ejemplo, "señalización por directorios" para localizar la mercancía en la tienda) y estimar la duración de las 
encuestas. Las declaraciones de algunos de los reactivos, elegidos al azar, fueron formuladas en forma negativa para prevenir la monotonía en el cuestionario y asegurar que el cliente esté leyendo y elaborando su juicio al responder a cada pregunta y no sólo repitiendo las respuestas que asignó a los primeros reactivos en cada sección.

La siguiente fase de la metodología fue la selección de la unidad de análisis. Se trabajó con una cadena de supermercados de cobertura regional, bien identificada por el mercado de consumidores y con múltiples unidades de negocio localizadas en la zona metropolitana de Toluca y Metepec. Esta cadena fue elegida principalmente por la disponibilidad e interés de la gerencia por apoyar este proyecto y, si bien tiene cobertura regional, se ha mantenido en el mercado haciéndole competencia a las grandes cadenas de supermercados de cobertura nacional e internacional. Se realizó una presentación de los objetivos y diseño del estudio a la gerencia para gestionar formalmente su participación. La gerencia de la cadena propuso las unidades de negocio (diez) en las cuales realizar el estudio. Los investigadores responsables visitaron cada unidad para presentar los objetivos del estudio con el gerente y negociar las fechas y condiciones de aplicación de la encuesta.

La tercera parte de la metodología fue el diseño del plan de muestreo. En el diseño del plan se utilizaron los datos históricos del número de clientes atendidos mensualmente en cada establecimiento, los cuales fueron proporcionados por la gerencia de la cadena. Se construyeron gráficos de series de tiempo para cada unidad, los datos disponibles cubren el periodo de enero de 2005 a abril de 2006. Todas las series exhiben un patrón horizontal, por lo que se aplicó el método de suavización exponencial simple para pronosticar el número de clientes que se esperaba atender durante el mes de septiembre de 2006, periodo elegido para aplicar la encuesta.

Cada tienda se trató como una unidad de muestreo independiente para seleccionar una muestra representativa durante una semana particular del mes de septiembre. El método de muestreo aplicado fue el muestreo sistemático, seleccionándose clientes a lo largo de varios días y horarios durante la semana. De acuerdo con el contenido de la encuesta, el tamaño de muestra se calculó tomando en cuenta los objetivos de la gerencia de cada tienda y del proyecto de investigación. La gerencia de cada tienda estableció como objetivo general la estimación del porcentaje de clientes globalmente satisfechos con el establecimiento (pregunta A.5) con un error de estimación no mayor del 5\%. En tanto que para el proyecto de investigación el objetivo fue estimar el índice medio de satisfacción del cliente con un margen de error no mayor a 0.2 unidades sobre la escala de cinco categorías empleada. Los resultados del premuestreo indicaron que alrededor del $70 \%$ de los entrevistados estaban satisfechos con el servicio de la tienda (respuestas inferiores a 3 para la pregunta A.5), siendo el promedio 
Construcción de un índice de satisfacción para clientes de supermercados mexiquenses

Una investigación exploratoria

de satisfacción del 2.01 y la desviación estándar de 1.04. Esta información se utilizó para calcular el tamaño de muestra bajo los dos objetivos:

a) Tamaño de muestra requerido para estimar la proporción de clientes satisfechos (respuestas menores de 3 a la pregunta A.5) con un margen de error de máximo 5\%.

b) Tamaño de muestra requerido para estimar el promedio de satisfacción global (pregunta A.5) con un margen de error de a lo más $5 \%$ (equivalente a 0.2 unidades en la escala de cinco categorías).

El tamaño de muestra para el caso (a) estuvo entre 134 a 136 clientes por tienda; para el caso (b) entre 106 y 107. Incorporando a estos límites las restricciones de tiempo y disponibilidad de encuestadores, se propuso el punto medio como tamaño de muestra más conveniente, lo cual corresponde a 120 encuestas por tienda.

Con el apoyo de la gerencia se determinaron los horarios y días de menor y mayor venta con la consideración de que el perfil de los clientes varía en estos periodos; esta información se utilizó para definir intervalos en los cuales seleccionar a los clientes. Un 70\% de los clientes (84 de los 120 a muestrear en cada unidad) fueron seleccionados durante los días de mayor venta, mientras que el $30 \%$ en los días de baja venta; además, dentro de cada día, 70\% de los clientes (60 personas en el caso de los días de alta venta) fueron entrevistados en horarios de alta venta y el restante $30 \%$ entrevistados en horarios de baja venta diaria. Este esquema de selección asegura que clientes con diversos perfiles participan en el estudio y toma en cuenta la alta incidencia de clientes en ciertos días y periodos dentro del día.

Las encuestas fueron aplicadas por estudiantes de la licenciatura en Mercadotecnia del ITESM, Campus Toluca. Los estudiantes fueron instruidos por los responsables de este estudio con respecto a los objetivos del proyecto, del contenido del cuestionario y de la forma en que deberían interactuar con los clientes para realizar la aplicación de la encuesta. Un total de 48 estudiantes fueron asignados a las distintas unidades de supermercados participantes; los estudiantes realizaron la encuesta durante una semana del mes de septiembre elegida de acuerdo con su disponibilidad y conveniencia. El total de encuestas planeadas (1200) se completaron durante la semana, no se registraron rechazos porque se contó con el apoyo de la gerencia de cada unidad para que cada cliente seleccionado aceptara responder a la encuesta.

A efecto de validar el trabajo de campo y preparar los datos para el análisis, se procedió a una revisión de las encuestas para verificar que estuviese completo el cuestionario y que hubiese congruencia en las respuestas. Un muy bajo porcentaje de 
cuestionarios no fueron aceptados (menos del $0.7 \%$ ) y en estos casos se regresó al campo para suplir las encuestas insatisfactorias. Una vez completado el número de encuestas para cada tienda, se realizó la codificación de las respuestas asignándose siempre valores bajos a respuestas favorables (más satisfacción o más lealtad intencional implica un valor de escala inferior), fijándose un código único para datos perdidos y otro para la categoría "no sabe"; los datos así editados se ingresaron a una hoja de cálculo. Posterior a la captura, se comprobó de nuevo la congruencia de las respuestas y se corrigieron los datos impropios antes de proceder con el análisis.

\section{Análisis y discusión de resultados}

Para contextualizar los hallazgos por presentar en esta sección, se describe el perfil demográfico de los clientes que conformaron la muestra de estudio. Las 1200 opiniones recopiladas corresponden principalmente a clientes mujeres, cuya edad oscila entre los 20 y los 50 años, dedicadas fundamentalmente al hogar o bien empleadas y cuyo ingreso medio se concentra en el rango de $\$ 2,500$ a menos de $\$ 9,999$. Este perfil general difiere para algunas de las unidades de negocio analizadas. La alta gerencia, en su revisión del reporte para esta investigación, validó el perfil de los clientes para cada una de las tiendas lo cual asegura la representatividad y calidad del muestreo realizado.

\section{Definición de dimensiones latentes para el concepto de satisfacción}

El primer análisis realizado sobre los datos fue un análisis factorial cuyo propósito fue evaluar la multidimensionalidad del concepto de satisfacción y revisar la estructura en cuatro componentes que se propuso a priori. Todos los análisis estadísticos se realizaron utilizando el paquete computacional spss, con la opción de manejo de valores perdidos por pareja con el fin de aprovechar la mayor parte de los datos en el cálculo de los coeficientes de correlación simple, que son las entradas de la matriz de correlación que se sometió al análisis factorial.

De los 32 reactivos iniciales que constituyen el índice de satisfacción, cinco fueron eliminados debido al alto porcentaje (más del 70\%) de respuestas de "no sabe" (ya sea porque el encuestado no tenía experiencia con el servicio o no contaba con información suficiente para evaluarlo). Los reactivos eliminados fueron: el sistema de apartado de artículos, el servicio de devolución de productos, el tiempo del servicio de entrega a domicilio, la habilidad del gerente para resolver los problemas reportados por los clientes y la respuesta del establecimiento a quejas presentadas.

Para asegurar la aplicabilidad del análisis factorial, se realizó la prueba de esfericidad de Bartlett, la cual resultó altamente significante $(\mathrm{P}=0.000)$ indicando que la matriz de correlación difiere de la idéntica y, por lo tanto, hay patrones de asociación entre 
Construcción de un índice de satisfacción para clientes de supermercados mexiquenses

Una investigación exploratoria

las respuestas a los reactivos lo que permite la identificación de variables latentes subyacentes. Se utilizó el método de componentes principales para realizar la extracción de factores. Todos los reactivos en las secciones B, C, D y E fueron incluidos, excepto los eliminados previamente por tener un porcentaje inaceptable de datos perdidos. Se aplicaron los criterios de scree-test, valores característicos (eigen-valores) mayores de uno y porcentaje de varianza explicada para determinar un número conveniente de factores por extraer. La figura 1 muestra el scree-plot resultante, el cual sugiere la presencia de uno a dos factores que explican la mayor parte de la varianza original, y de hasta siete factores con varianzas (eigen-valores) superiores a uno. La solución en siete factores explica el $56.3 \%$ de la varianza original. Si bien este porcentaje no es muy elevado, la estructura de la solución después de la rotación resulta satisfactoria para fines de interpretación, por lo tanto, la elegida para definir el índice de satisfacción con el servicio de supermercados.

Figura 1

Scree-plot para el análisis factorial de los reactivos de satisfacción

\section{Scree Plot}

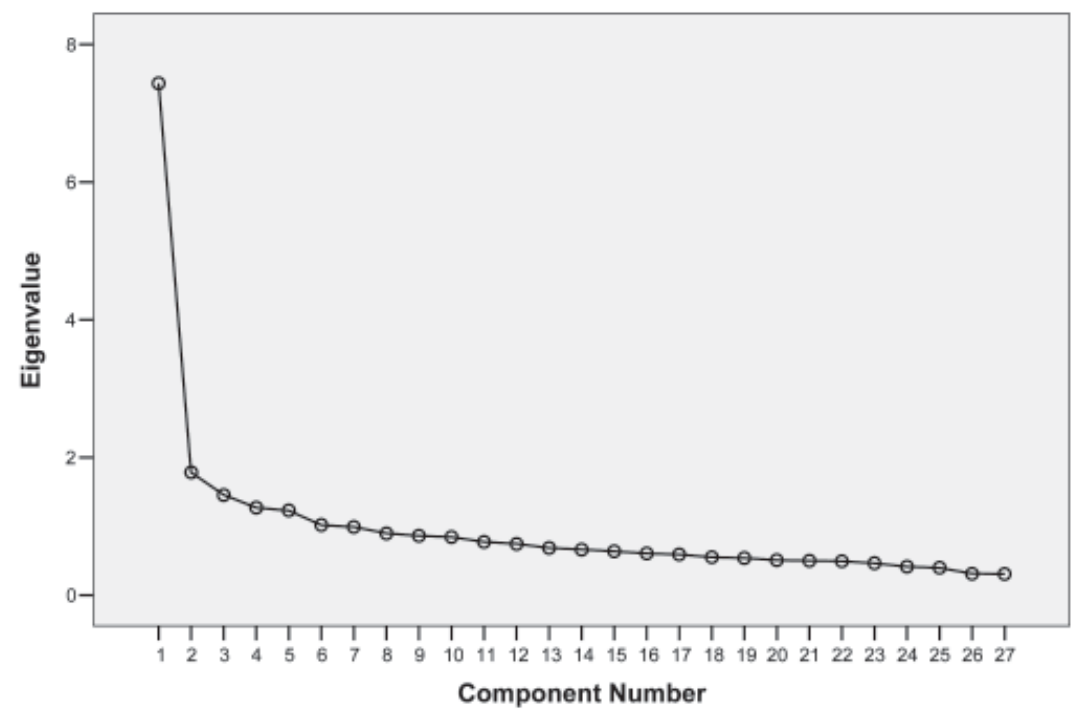

Se aplicó una rotación Varimax a la matriz de cargas inicial, obteniéndose convergencia después de ocho iteraciones. Todos los factores incluyen más de tres reactivos, excepto por el último extraído, lográndose una buena distinción entre los factores en 
el sentido de que la mayoría de los reactivos tuvieron cargas diferencialmente mayores en un solo factor. Para la interpretación de la solución, se declararon altamente significantes todas aquellas cargas superiores a $2(0.081)=0.162$ en valor absoluto (Stevens, 1986: 344). Varios reactivos resultaron con cargas significantes en más de un factor, asignándose a aquel factor en el cual exhibían la mayor carga y en el cual se agrupaban con otros reactivos que a priori formaron parte de la misma sección o componente de satisfacción. Se probó con rotaciones oblicuas para explorar si se obtenía una estructura en la cual más reactivos tuvieran cargas significantes en un solo factor; sin embargo estas alternativas de rotación no mostraron una ventaja sobre la rotación Varimax, por lo que se optó por interpretar la matriz de componentes rotadas desde esta alternativa.

En la tabla 1 se describe el contenido de cada uno de los siete factores extraídos, el cual se asocia con un componente latente de la satisfacción del cliente de supermercados. La primera columna de la tabla propone un nombre genérico que identifica al componente, la segunda columna resume el contenido de los reactivos que se asignan a este componente y la tercera muestra las cargas correspondientes a cada reactivo dentro del factor.

Tabla 1

Componentes propuestos para el Î́ndice de Satisfacción del Consumidor de Supermercados y Carga de los Factores

\begin{tabular}{|c|c|c|}
\hline $\begin{array}{l}\text { Componentes de } \\
\text { satisfacción }\end{array}$ & Operacionalización de los componentes & Cargas \\
\hline & & Componente 1 \\
\hline \multirow{6}{*}{ Empleados } & Competencia de los empleados & 0.721 \\
\hline & Actitud de los empleados & 0.761 \\
\hline & $\begin{array}{l}\text { Competencia del gerente para dar un buen servicio y atender los } \\
\text { requerimientos de los clientes }\end{array}$ & 0.615 \\
\hline & Cajeras & 0.643 \\
\hline & Percepción general del servicio de los empleados & 0.712 \\
\hline & & Componente 2 \\
\hline \multirow[t]{6}{*}{ Mercancía } & Calidad de los productos & 0.681 \\
\hline & Variedad de categorías de productos & 0.797 \\
\hline & Variedad de marcas & 0.775 \\
\hline & Frescura de productos perecederos & 0.618 \\
\hline & Existencia de productos favoritos & 0.640 \\
\hline & & Componente 3 \\
\hline \multirow{5}{*}{$\begin{array}{l}\text { Servicios } \\
\text { determinantes }\end{array}$} & Horarios & 0.494 \\
\hline & Ubicación & 0.659 \\
\hline & Formas de pago aceptadas & 0.705 \\
\hline & Promociones de la tienda & 0.390 \\
\hline & Ambiente de la tienda & 0.535 \\
\hline
\end{tabular}


Construcción de un índice de satisfacción para clientes de supermercados mexiquenses

Una investigación exploratoria

\begin{tabular}{lll}
\hline \multirow{2}{*}{ Valor percibido } & Componente 4 \\
& Relación precio/servicio & 0.368 \\
& Relación precio/ambiente de la tienda & 0.811 \\
& Relación precios de la tienda/precios de la competencia & 0.799 \\
\hline \multirow{2}{*}{$\begin{array}{l}\text { Tangibles } \\
\text { complementarios }\end{array}$} & Limpieza de la tienda & Componente 5 \\
& Servicio de estacionamiento & 0.257 \\
& Seguridad de las instalaciones & 0.803 \\
Layout & & 0.636 \\
& Apariencia general de la tienda & Componente 6 \\
& Pasillos & 0.372 \\
& Localización de los departamentos & 0.703 \\
& Señalización & 0.436 \\
Insuficiencia & & 0.512 \\
del servicio & Tiempo de espera en cajas & Componente 7 \\
\hline
\end{tabular}

De los siete factores, tres corresponden a componentes propuestos a priori y que además se han citado recurrentemente en la literatura. Estas variables latentes se identifican con el servicio prestado por los empleados (Kristensen et al., 2001 denominan en su modelo a este componente como Human-ware); la calidad y variedad de la mercancía que oferta la tienda (componente considerado en los índices de satisfacción ECSI y ACSI) y valor percibido en el sentido de la relación precio/servicio; este componente fue considerado en los modelos ECSI y ACSI como variable moderadora entre los componentes de empleados y mercancía y la satisfacción del cliente. La correspondencia entre la estructura a priori y la obtenida después del análisis factorial es evidencia de validez convergente para el índice de satisfacción elaborado.

Los factores restantes (cuatro) resultan del reagrupamiento de los reactivos que inicialmente constituían la sección c, referente a las instalaciones y servicios de apoyo de la tienda. Los nuevos factores se identificaron como sigue:

F3 = Servicios determinantes. Este factor describe aspectos fundamentales para el cliente que tienen que ver con los horarios, ubicación y facilidades de compra que oferta la tienda. Ubicación y horario son dos elementos críticos en el sector de supermercados de acuerdo con la gerencia de la tienda participante, y también de acuerdo con la literatura disponible para el sector, por ejemplo, McDonald (1991) identifica a la ubicación como el elemento más importante para la satisfacción.

F5 = Tangibles complementarios. En este factor se agrupan los reactivos relacionados con la limpieza en la tienda, la disponibilidad de estacionamiento y la seguridad de sus instalaciones. Si bien éstos no son aspectos centrales al servicio de un supermer- 
cado, son apreciados por los clientes en el momento de dar juicios de satisfacción. Este tipo de tangibles complementarios se ha identificado como un elemento que incide en la lealtad del cliente de tiendas de venta al menudeo (Al-Awadi, 2002).

F6 = Layout. El factor cubre aquellos elementos que tienen que ver con la distribución de los departamentos en la tienda, la señalización, la apariencia del establecimiento y ancho de los pasillos. En el contexto de supermercados, este factor fue también identificado como determinante para la lealtad de los clientes por autores como Sirohi et al. (1998).

F7 = Insuficiencia en el servicio. Este factor sólo agrupa a dos reactivos, uno referente a los tiempos de espera en cajas y el otro a la percepción sobre la suficiencia del número de empleados para atender a la clientela. El tiempo de espera en cajas se ha mostrado como influyente sobre la satisfacción (Gail y Scott, 1995) y en nuestro estudio estuvo altamente asociado con el número de empleados porque los clientes consideran que parte de la espera en cajas se debe a que hay pocos empleados disponibles para realizar el cobro o aclarar dudas de precio en cajas.

Es importante precisar que la distribución de reactivos en los cuatro factores antes descritos no empata completamente con la literatura a excepción de los correspondientes al Factor 6 titulado Layout, cuyos reactivos son paralelos a los del componente nombrado como Apariencia de la tienda por Sirohi et al. (1995). El agrupamiento de los demás reactivos determinantes de la satisfacción claramente muestra que los consumidores distinguen entre lo que constituye el servicio básico que da un supermercado y los servicios secundarios. En otros estudios, estos servicios complementarios -en particular el estacionamiento y el tiempo de espera en cajas- se han integrado dentro del servicio básico, lo cual atribuimos a necesidades diferentes de los clientes en otros contextos. En este estudio, la mayoría de los participantes fueron individuos de nivel socioeconómico medio-bajo para quienes el estacionamiento o la espera en cajas no resultan ser servicios prioritarios.

\section{Análisis de confiabilidad interna para el índice de satisfacción}

Para evaluar la fiabilidad del índice de satisfacción elaborado, se procedió al cálculo del coeficiente alfa de Cronbach para cada dimensión o factor identificado. Los resultados correspondientes a este análisis de confiabilidad se muestran en la tabla 2, sólo dos de los siete coeficientes, aquellos asociados con los primeros factores son superiores a 0.7 , valor que se ha tomado como cota inferior de referencia para declarar una buena confiabilidad interna. El promedio de los primeros seis coeficientes -el último corresponde a un factor que sólo contiene dos reactivos- es de 0.656 valor que tam- 
Construcción de un índice de satisfacción para clientes de supermercados mexiquenses

Una investigación exploratoria

poco excede la cota inferior de 0.7 , por lo cual la confiabilidad de la multiescala de satisfacción no es globalmente satisfactoria. En un esfuerzo de mejorar la confiabilidad de la escala se calcularon los coeficientes de correlación simples entre cada uno de los reactivos que incluye cada factor y el total correspondiente. En la última columna de la tabla 2 se reportan estos coeficientes, indicándose entre paréntesis aquel reactivo individual para el cual el coeficiente de correlación es inferior a 0.5. Después de eliminar los reactivos con bajos coeficientes de correlación con el total, se recalculó el coeficiente de confiabilidad (valor entre paréntesis en la columna con la leyenda Alfa de Cronbach) sin lograr una mejora en el valor de los coeficientes debido a la reducción en el número de reactivos para cada dimensión subyacente excepto la de Mercancía. Debido a esta situación, la confiabilidad de la multiescala de satisfacción no puede garantizarse a pesar de que fue elaborada empleando como referencia escalas validadas en otros contextos. La transferencia y aplicación de escalas, calificadas como confiables y válidas en la literatura especializada, presenta problemas cuando se utilizan para evaluar las percepciones de los consumidores mexicanos (Trujillo y Arroyo, 2006), cuyas apreciaciones parecen ser menos racionales e integradas que las de consumidores de distinta idiosincrasia que durante más tiempo han podido elegir entre múltiples opciones que compiten por su preferencia.

Tabla 2

Cálculo de índices de confiabilidad, Alfa de Cronbach

\begin{tabular}{lccc} 
Variable & $\begin{array}{c}\text { Número } \\
\text { de reactivos }\end{array}$ & $\begin{array}{c}\text { Valor del } \\
\text { Alfa }\end{array}$ & $\begin{array}{c}\text { Coeficientes de correlación } \\
\text { con el puntaje factorial }\end{array}$ \\
\hline Empleados & 5 & $\begin{array}{c}0.778 \\
(0.686)\end{array}$ & $0.066(\mathrm{D} 25), 0.727,0.757,0.41(\mathrm{D} 30), 0.640$ \\
\hline Mercancía & 5 & 0.805 & $0.693,0.789,0.774,0.608,0.627$ \\
\hline $\begin{array}{l}\text { Servicios } \\
\text { determinantes }\end{array}$ & 5 & $\begin{array}{c}0.657 \\
(0.637)\end{array}$ & $0.502,0.691,0.395(\mathrm{C} 14), 0.683,0.527$ \\
\hline Valor percibido & 3 & $\begin{array}{c}0.598 \\
(0.610)\end{array}$ & $0.343(\mathrm{~B} 6), 0.821,0.8$ \\
\hline $\begin{array}{l}\text { Tangibles } \\
\text { complementarios }\end{array}$ & 3 & $\begin{array}{c}0.566 \\
(0.493)\end{array}$ & $0.370(\mathrm{C} 16), 0.822,0.622$ \\
\hline Layout & 4 & 0.536 & $0.382(\mathrm{C} 15), 0.687,0.476(\mathrm{C} 18), 0.562$ \\
\hline $\begin{array}{l}\text { Insuficiencia del } \\
\text { servicio }\end{array}$ & 2 & $0.253)$ & $0.673,0.261$ \\
\hline
\end{tabular}

Previo al análisis para determinar si el índice de satisfacción predice las intenciones de lealtad de los clientes, se aplicó también un análisis factorial a los tres reactivos que forman la sección $\mathrm{F}$ del cuestionario. Bajo los criterios de valores característicos superiores a uno y scree-plot, un solo factor fue extraído, lo cual es totalmente congruente con lo establecido en la literatura garantizando la validez convergente de la multiescala de tres reactivos diseñada para medir lealtad intencional. Esta solución en 
un único factor explica el $43.4 \%$ de la varianza original y las cargas de los reactivos son todas altamente significantes, estando en el rango de 0.405 a 0.772 .

\section{Validación predictiva para el índice de satisfacción}

Múltiples estudios dan apoyo empírico a la relación causal entre la satisfacción y la lealtad, si bien la satisfacción es una condición necesaria no es suficiente para asegurar un comportamiento leal por parte del cliente (Oliver, 1999). Para apoyar la validez del índice de satisfacción elaborado en el sentido de que éste prediga otros conceptos consecuentes, se aplicó un análisis de regresión múltiple. En este análisis, la variable dependiente es el puntaje factorial calculado a partir de los tres reactivos de lealtad y las variables independientes corresponden a los puntajes factoriales asociados a las tres dimensiones de satisfacción (Empleados, Mercancía y Servicios determinantes) para los cuales el índice de confiabilidad interna es satisfactorio, esto es cercano o superior a 0.7. Los puntajes factoriales fueron calculados directamente con SPSS a través de la rutina de análisis factorial; cabe aclarar que en esta rutina cuando hay un dato perdido el puntaje factorial ya no se calcula. Para evitar que una gran parte de los datos no entraran al análisis debido a que un único reactivo tenía una respuesta faltante, se optó por sustituir los datos perdidos por el promedio correspondiente. Esta sustitución se realizó únicamente cuando en una cierta variable el porcentaje de datos perdidos fuera inferior al 5\%; además, el promedio por el cual se sustituyeron los datos perdidos no fue el promedio global para la variable, sino el promedio de las respuestas disponibles para una misma unidad de negocios. De esta forma, se toman en cuenta las diferencias en las evaluaciones de los clientes de tienda en tienda.

El modelo de regresión lineal múltiple ajustado resultó altamente significante ( $\mathrm{F}=$ 91.147 y $\mathrm{P}=0.000$ en el ANOva) con un coeficiente de determinación ajustado de 0.232 , el cual indica que un $23.2 \%$ de la varianza en las intenciones de lealtad de los clientes se explica a través de sus niveles de satisfacción para distintos aspectos o dimensiones de la tienda. El valor de este coeficiente de determinación refleja el hecho de que la satisfacción no sea el único elemento que determina las intenciones de lealtad. En la tabla 3 se muestran los coeficientes de regresión no-estandarizados y estandarizados (valores beta). Las tres dimensiones consideradas tienen coeficientes de regresión significantes, todos ellos positivos, lo cual indica — dada la codificación de datos utilizada - que un incremento en alguno de los tres componentes de satisfacción (percepción más desfavorable) conduce a un incremento en el puntaje de lealtad (menores intenciones de ser leal a la tienda). 
Construcción de un índice de satisfacción para clientes de supermercados mexiquenses

Una investigación exploratoria

Tabla 3

Análisis de regresión lealtad vs. dimensiones latentes de satisfacción

\begin{tabular}{|c|c|c|c|c|c|}
\hline \multirow[t]{2}{*}{ Modelo } & \multicolumn{2}{|c|}{$\begin{array}{l}\text { Coeficientes no } \\
\text { estandarizados }\end{array}$} & $\begin{array}{c}\text { Coeficientes } \\
\text { estandarizados }\end{array}$ & $\mathbf{t}$ & Sig. \\
\hline & Coeficiente de regresión & Error estándar & Beta & & \\
\hline (Constante) & -.033 & .029 & & -1.122 & .262 \\
\hline Empleados & .257 & .029 & .259 & 8.84 & .000 \\
\hline Mercancía & .346 & .030 & .336 & 11.460 & .000 \\
\hline $\begin{array}{l}\text { Servicios } \\
\text { determinantes }\end{array}$ & .220 & .030 & .217 & 7.384 & .000 \\
\hline
\end{tabular}

En congruencia con estudios precedentes, los tres componentes de satisfacción asignados a los tres primeros factores tienen un efecto significante sobre las recomendaciones e intenciones de continuar comprando en la tienda aun cuando ésta se reubicara más lejos o se incrementara la oferta de supermercados en la zona. Con base en el valor de los coeficientes de regresión estandarizados se establece que la dimensión de satisfacción más importante es la oferta de mercancía del supermercado. En un segundo término figura el servicio y atención prestados por los empleados de la tienda, seguido de los servicios determinantes que se espera de un supermercado; esto es, horarios convenientes, ubicación, formatos de pago, etcétera.

\section{Conclusiones y recomendaciones}

El concepto de satisfacción es un concepto que involucra múltiples dimensiones que cubren no sólo los aspectos relacionados con el resultado del servicio; es decir, la adquisición de mercancía en tiempos y localizaciones convenientes al consumidor, sino también aspectos referentes a la atención y apoyo de empleados, y el lograr un ambiente y acomodo de mercadería que facilite y haga grata la compra. Este estudio aporta apoyo empírico para la confiabilidad, validez facial, convergente y predictiva de una multiescala de satisfacción para el cliente mexiquense de supermercados que puede implantarse como medida adicional del desempeño de este tipo de establecimientos.

El índice de satisfacción elaborado en este trabajo incluye sólo tres dimensiones latentes, las cuales reproducen componentes recurrentemente identificados en la literatura: calidad y variedad de la mercancía, atención y capacidad de empleados y servicios determinantes como ubicación y horarios del establecimiento. Los otros cuatro componentes -identificados en el análisis factorial aplicado al índice- resultaron con confiabilidad interna inaceptable aun cuando la literatura los reporta como factores que influyen en la satisfacción o lealtad de los clientes de supermercados. Este resultado revela la importancia de realizar adaptaciones a escalas validadas en otros 
contextos antes de aplicarlas en otro ambiente, pues es necesario que las escalas que se transfieren tomen en cuenta la idiosincrasia del consumidor mexicano. Durante la aplicación de las encuestas, se identificó que el cliente de supermercados en el estrato medio-bajo hace una distinción entre los servicios primarios del supermercado y aquellos servicios y tangibles que no le resultan prioritarios (estacionamiento, tiempo de espera en cajas, ancho de pasillos, entre otros).

Las percepciones de satisfacción de los clientes determinan sus intenciones de lealtad hacia los supermercados mexiquenses de acuerdo con el análisis de regresión efectuado en este trabajo. En consecuencia, estos establecimientos han de procurar satisfacer los requerimientos básicos de su clientela con el fin de contrarrestar la entrada de otros establecimientos similares y aprovechar las recomendaciones favorables de los clientes para fortalecer su imagen. Los componentes de satisfacción que inciden significativamente sobre la lealtad intencional de los clientes son, en orden de importancia: calidad y variedad de la mercancía, atención y capacidad de los empleados y servicios básicos como acceso, tiempo y formas de pago. Estos componentes se identifican como los calificadores esenciales para los supermercados. Cuando un establecimiento no es capaz de satisfacer estos elementos básicos y recrear la experiencia de compra, no puede competir por mercado. Las otras cuatro dimensiones latentes del índice - valor percibido, tangibles complementarios, layout e insuficiencias específicas en servicio relacionadas con espera en cajas y número de empleados que atienden- no calificaron como confiables. Esto no quiere decir que no sean componentes de la satisfacción, sino que tienen que reformularse después de considerar las características culturales, psicológicas y económicas del consumidor mexicano a través de una exploración a fondo con métodos cualitativos.

Las recomendaciones positivas sobre el supermercado y la intención de incurrir en costos de traslado en caso de reubicación de la tienda fueron las intenciones específicas de lealtad más fuertemente influidas por las evaluaciones de satisfacción. La intención de seguir comprando en el supermercado en caso de que tiendas similares se instalaran en la zona no resultó afectada en la misma intensidad por las evaluaciones de satisfacción. Esto sugiere que a pesar de la evaluación satisfactoria de la clientela para la cadena de supermercados en estudio, el cliente está dispuesto a probar nuevas opciones y tener variedad para sus compras. Por lo que más que una lealtad auténtica, lo que el supermercado puede lograr es una preferencia a nivel de lealtad cognitiva y el que forme parte del conjunto de consideraciones (consideration set) del cliente.

Este estudio se realizó para una cadena de supermercados de cobertura regional; los resultados muestran que la estructura de los índices de satisfacción empleados para cadenas de mayor cobertura en ambientes — donde este formato de compra y tienda 
Construcción de un índice de satisfacción para clientes de supermercados mexiquenses

Una investigación exploratoria

tiene ya un arraigo de muchos años- no pueden ser replicados sin adaptaciones. Una extensión a este trabajo es modificar el índice propuesto y validarlo en múltiples cadenas regionales y locales con el fin de asegurar su validez como medida de satisfacción para el cliente mexicano que prefiere comprar en establecimientos donde se oferta mercancía particular a su subcultura. Otras extensiones a esta investigación son: contrastar los componentes de satisfacción para el consumidor de supermercados regionales versus aquellos de cobertura nacional e internacional y tomar en cuenta las características de los consumidores, así como contrastar sus expectativas y niveles de satisfacción de acuerdo con su perfil. También resulta interesante analizar el efecto que sobre la lealtad tienen variables adicionales a la satisfacción, tales como involucramiento con la compra, tipo de establecimiento de venta al menudeo (hipermercado, supermercado, tienda de conveniencia, etcétera) e influencias externas para la compra como es la intensidad de la publicidad.

\section{Bibliografía}

Al-Awadi, A. (2002), "A proposed model of consumer loyalty in the retailing sector based on Kuwaiti experience”, Total Quality Management, 13, 7, pp. 1035-1046.

Bloemer, J. y K. Ruyter (1998), "On the relationship between store image, store satisfaction and store loyalty", European Journal of Marketing, 32, 5/6, pp. 499-513.

Chang, C.-H. y C.-Y. Tu (2005), "Exploring store image, customer satisfaction and customer loyalty relationship: evidence from taiwanese hypermarkets industry", The Journal of American Academy of Business, 7, 2, pp. 197-202.

Fornell, C., M. Johnson, E. Anderson, J. Cha y B. Bryant (1996), "The american customer satisfaction index: nature, purpose and findings", Journal of Marketing, 60, 4, pp. 7-18.

Gail, T. y L. Scotт (1995), "Waiting time delays and consumer satisfaction in supermarkets", The Journal of Services Marketing, 9, 5, pp. 20-29.

Kristenensen, K., H. Juhl y P. Ostergaard (2001), "Customer satisfaction: some results for european retailing”, Total Quality Management, 12, 7/8, pp. 890-897.

McDonald, G. (1991), "The influence of supermarket attributes on perceived customer satisfaction: an East Asian study", International Review of Retail, Distribution and Consumer Research, 1, 3, pp. 315-327.

Oliver, R. (1999), “Whence consumer loyalty?”, Journal of Marketing, 63, pp. 33-44. 
SAwmong, S. y O. Omar (2004), "The store loyalty of the UK's retail consumers", Journal of American Academy of Business, 5, 1/2, pp. 503-509.

Stevens, J. (1986), Applied Multivariate Statistics for the Social Sciences, Lawrence Erlbaum Associates, Inc., Hillsdale.

Sirohi, N., E. McLaughlin y D. Wittink (1998), "A model of consumer perceptions and store loyalty intentions for a supermarket retailer", Journal of Retailing, 74, 2, pp. 223-245.

Spreng, R., S. MacKenzie y R. Olshavsky (1996), “A reexamination of the determinants of consumer satisfaction", Journal of Marketing, 60, pp. 15-32.

SweEney, J. y G. Soutar (2001), "Consumer perceived value: the development of a multiple item scale", Journal of Retailing, 77, pp. 203-220.

Trujillo León, A. y P. E. Arroyo López (2006), "Evaluación del impacto de factores de diseño de un restaurante sobre la lealtad", Memorias del 36 . Congreso de Investigación y Desarrollo del ITESM, tomo II, Toluca, Méxíco, pp. 95-103. 